\section{Mutación del gen BRAF en pacientes con cánceres de colon y recto con KRAS no mutado}

\author{
IVÁN ROA ${ }^{1}$, ANAKAREN GAME ${ }^{2, a}$, \\ CAROLINA BIZAMA ${ }^{1}$, KURT SCHALPER ${ }^{3}$
}

\section{BRAF gene mutation in wild-type KRAS patients with colorectal cancers}

\begin{abstract}
Background: In colorectal cancer, BRAF and KRAS mutation are mutually exclusive, but both are independent prognostic factors for the disease. Aim: To determine the frequency of BRAF V600E mutation in colorectal cancer. Material and Methods: A KRAS mutation study was carried out in 100 tissue samples of primary and metastatic adenocarcinomas of colon and rectum from patients aged $61.1 \pm 62$ years (56 women). Negative KRAS mutation cases underwent study of BRAF V600E mutation by restriction fragment length polymorphism (RFLP) and direct sequencing. Results: Primary tumors were located in the colon and rectum in 88 and six cases respectively. Five were liver metastases and in one case, the sample location was undetermined. Forty two samples were KRAS positive (mutated). In 12 of the 58 KRAS negative (wild type) samples, the V600E mutation in codon 15 of the BRAF gene was demonstrated. No differences in the frequency and distribution of mutations, stratified by gender, age, primary tumor versus metastasis, or tumor location were observed. Conclusions: Twelve percent of KRAS negative colorectal cancer samples showed BRAF gene mutation. Considering that $42 \%$ of samples have a KRAS mutation, 54\% of patients should not respond to therapies with monoclonal antibodies directed against epidermic growth factor (EGFR) pathway.
\end{abstract}

(Rev Med Chile 2014; 142: 55-60)

Key words: Colonic neoplasms; Mutation; Proto-Oncogene Proteins B-raf; Proto-Oncogene Proteins p21 (ras).

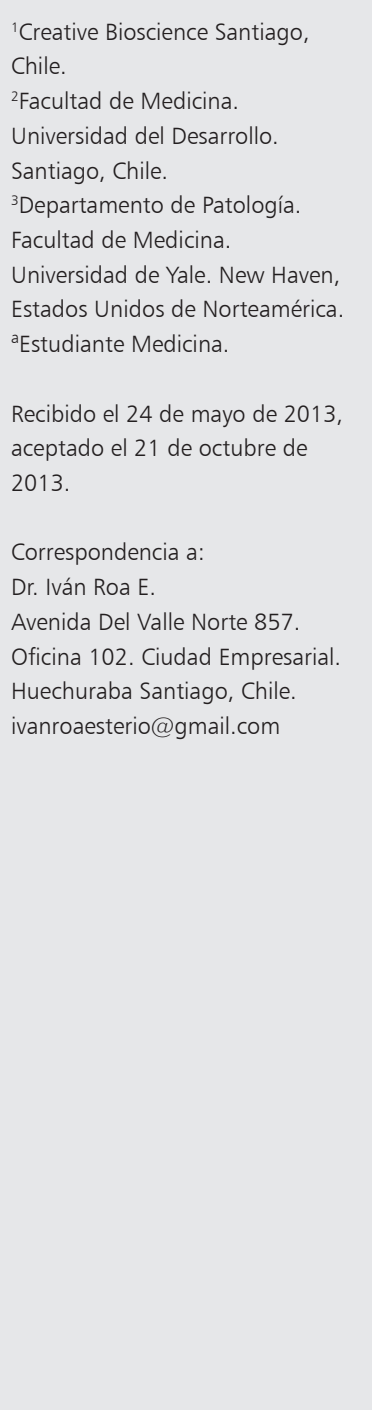

$\mathrm{E}$ 1 cáncer de colon y recto es el cuarto cáncer de mayor incidencia y mortalidad en el mundo y representa la tercera causa de mortalidad por tumores malignos en el hombre y el quinto en la mujer ${ }^{1}$. En Chile ocupa el tercer lugar entre los cánceres digestivos y el séptimo a nivel global con una mortalidad de alrededor de 8 por $10^{6}$ habitantes ${ }^{2-4}$. Desde el punto de vista genético-molecular, esta neoplasia ha sido extensamente caracterizada tanto en sus formas esporádicas como las hereditarias poliposas y no poliposas $^{5-7}$, así como los genes que participan y vías metabólicas involucradas ${ }^{8-10}$.
En los estadios avanzados en presencia de metástasis, la sobrevida global a 5 años es de menos de $15 \%$, a pesar de los avances de la quimioterapia $^{11}$. Los anticuerpos monoclonales, cetuximab y panitumumab, que tienen como diana al receptor del factor de crecimiento epidérmico (EGFR), han sido aprobados para el tratamiento de cáncer de colon y recto metastásico ${ }^{12-14}$. Sin embargo, sólo un porcentaje de estos pacientes son beneficiados con esta terapia debido a un alto porcentaje de pacientes no respondedores que pueden alcanzar sobre $40-60 \%{ }^{12-14}$. Entre los factores más importantes que determinan resistencia a los anticuerpos 
monoclonales está la mutación del gen KRAS, que se encuentra presente en alrededor de $40 \%$ de estos cánceres ${ }^{12,15}$. Las mutaciones de KRAS se localizan en su gran mayoría en sitios específicos como son los codones 12 y 13 y en menor frecuencia en el 61. Estas mutaciones son activantes, es decir, determinan que la vía corriente abajo se encuentre en continua y permanente actividad, estimulando la proliferación celular independiente del bloqueo de uno de los más potentes estimuladores de esta vía, como es el EGFR ${ }^{16}$. Pacientes cuyas células tumorales presentan KRAS mutado no tendrían indicación de bloqueadores o inhibidores del EGFR $^{17}$. Otros genes como BRAF también son parte de esta misma vía metabólica corriente abajo y mutaciones tendrían un efecto similar a KRAS mutado $^{12,18}$.

Mutaciones del gen BRAF han sido reportados entre $5-15 \%$ de los carcinomas de colon y recto ${ }^{19,20}$. La mutación de BRAF se observa casi exclusivamente en tumores esporádicos y está fuertemente asociado con inestabilidad microsatelital producto de la metilación del promotor del gen reparador del ADN MLH1 $1^{21,22}$. Un hecho demostrado es que la mutación de KRAS y BRAF son mutuamente excluyentes, es decir, cuando uno de estos genes presenta una mutación el otro excepcionalmente está mutado, por lo cual, ambos eventos pertenecientes a la vía RAS-RAF-MEK-ERK-MAP quinasa han sido sólo excepcionalmente reportados en menos de $0,02 \%$ de los casos en que ambos genes han sido estudiados simultáneamente ${ }^{23}$. Mutaciones del gen BRAF también se ha identificado en otros tumores malignos como el carcinoma papilar de tiroides, melanoma, adenocarcinomas del ovario y leucemias, entre otros ${ }^{24}$.

BRAF es un miembro de la familia RAF quinasa que contiene residuos serina/treonina que participan en las vías de señalización que regulan la proliferación, diferenciación y la apoptosis ${ }^{25,26}$. Hasta el momento, todas las mutaciones de BRAF han sido reportadas dentro de los dos dominios de quinasas y sobre $90 \%$ corresponden a una transversión de timina a adenina en la posición nucleotídica 1796, que convierte a la valina en glutamato. Esta mutación V600E previamente había sido designada como BRAF V599 $\mathrm{E}^{27,28}$.

La mutación de KRAS es un factor predictivo de la resistencia al tratamiento con anticuerpos anti-EGFR y se asocia con un mal pronóstico y a una menor supervivencia ${ }^{29}$. Por otro lado, la ausencia de mutación de KRAS no garantiza los beneficios de la terapia, ya que otros efectores corrientes abajo de KRAS pudiesen participar en esta resistencia. Se ha demostrado que la mutación V600E de BRAF, disminuye la respuesta a cetuximab o panitumumab en pacientes con cáncer de colon y recto metastático ${ }^{12,29,30}$. Se debe señalar que cuando KRAS o BRAF se encuentran mutados, alrededor de 55\% de los pacientes pueden tener la condición de no respondedores a la terapia anti EGFR, por lo que se ha sugerido recientemente la conveniencia de determinar el status de ambos genes previo al inicio de la terapia anti EGFR objetivo de este trabajo es determinar la frecuencia y distribución de las mutaciones del gen BRAF en el cáncer de colon y recto en nuestro país.

\section{Material y Método}

\section{Casos}

Se incluyen en este estudio de 100 adenocarcinomas primarios en estadios, avanzados y metástasis de adenocarcinomas de colon, recto. Las muestras provenientes de tejidos fijados en formalina e incluidos en parafina a las cuales se les había realizado estudio de mutación del gen KRAS y que demostraron una positividad en 42 casos (Roa et al datos no publicados). Las mutaciones de KRAS y BRAF al ser prácticamente excluyentes ${ }^{23}$, hace posible asegurar que la presencia de una descarta a la otra. De esta manera, se realizó estudio de la mutación BRAF V600EA exclusivamente a los 58 casos restantes, asumiendo que los 42 casos KRAS mutados eran BRAF de tipo silvestre o no mutado. Se confeccionó una base de datos anónima de las muestras, en la cual fueron consignados exclusivamente los siguientes parámetros: género, edad y localización del tumor. La metodología utilizada se puede resumir de la siguiente manera.

\section{Protocolo de extracción cuantificación y amplificación de ADN}

En los cortes seleccionados que incluían áreas con una población de al menos 30\% de células tumorales se realizó la desparafinización en baños de xilol e hidratación en concentraciones decrecientes de alcoholes hasta el agua y mantención de las muestras en solución tamponada. Posterior disección de las áreas seleccionadas. Los fragmentos de tejido obtenidos fueron resuspendidos y 
Mutación gen BRAF en cáncer de colon y recto - I. Roa et al

centrifugados por 5 min a $13.000 \mathrm{rpm}$. La extracción de ADN se realizó utilizando el kit AxyPrep Multisource Genomic DNA Miniprep kit Axygen ${ }^{\circledR}$ de acuerdo a las instrucciones del fabricante. La determinación de la integridad del ADN se realizó a través de la amplificación del gen de la $\beta$-globina con productos de amplificación esperados de 110 y $268 \mathrm{pb}$. La verificación de los productos se realizó por electroforesis en geles de agarosa a $2 \%$.

\section{Amplificación exón 15 gen BRAF}

Se utilizaron los siguientes partidores del exón 15 con productos esperados de 164pb: BRAF exon15:F 5'TCATAATGCTTGCTCTG ATAGGA3', exon 15:R 3'GGCCAAAAATTTAATCAGTGGA5'. Las condiciones del PCR fueron una denaturación inicial a $95^{\circ} \mathrm{C}$ por $5 \mathrm{~min}$, seguido de 40 ciclos de denaturación a $95^{\circ} \mathrm{C}$ por 30 segundos, alineación a $56{ }^{\circ} \mathrm{C}$ por 30 segundos, elongación a $72{ }^{\circ} \mathrm{C}$ por
30 segundos y una extensión final a $72{ }^{\circ} \mathrm{C}$ por 5 min. Los productos del PCR fueron visualizados en geles de Agarosa a $4 \%$ y teñidos con bromuro de etidio.

\section{RFLP (Restriction Fragment Length Polimorphism)}

Se realizó PCR semianidado con los siguientes partidores: F 5 CAT AAT GCT TGC TCT GAT AGG A 3`y R 5`CAG ACA ACT GTT CAA ACT GAT G 3' con un producto esperado de $140 \mathrm{pb}$. La digestión se realizó con enzimas de restricción Hpy166II para el sitio de corte en presencia de la secuencia no mutada (ACA GTG AAA) del codón 15 con formación de dos fragmentos de $121 \mathrm{pb}$ y $19 \mathrm{pb}$. En presencia de la mutación (ACA GAG AAA) no se produce el corte. La visualización de los productos se realizó en geles de agarosa a 3\% teñidos con bromuro de etidio (Figura 1a - 1b).

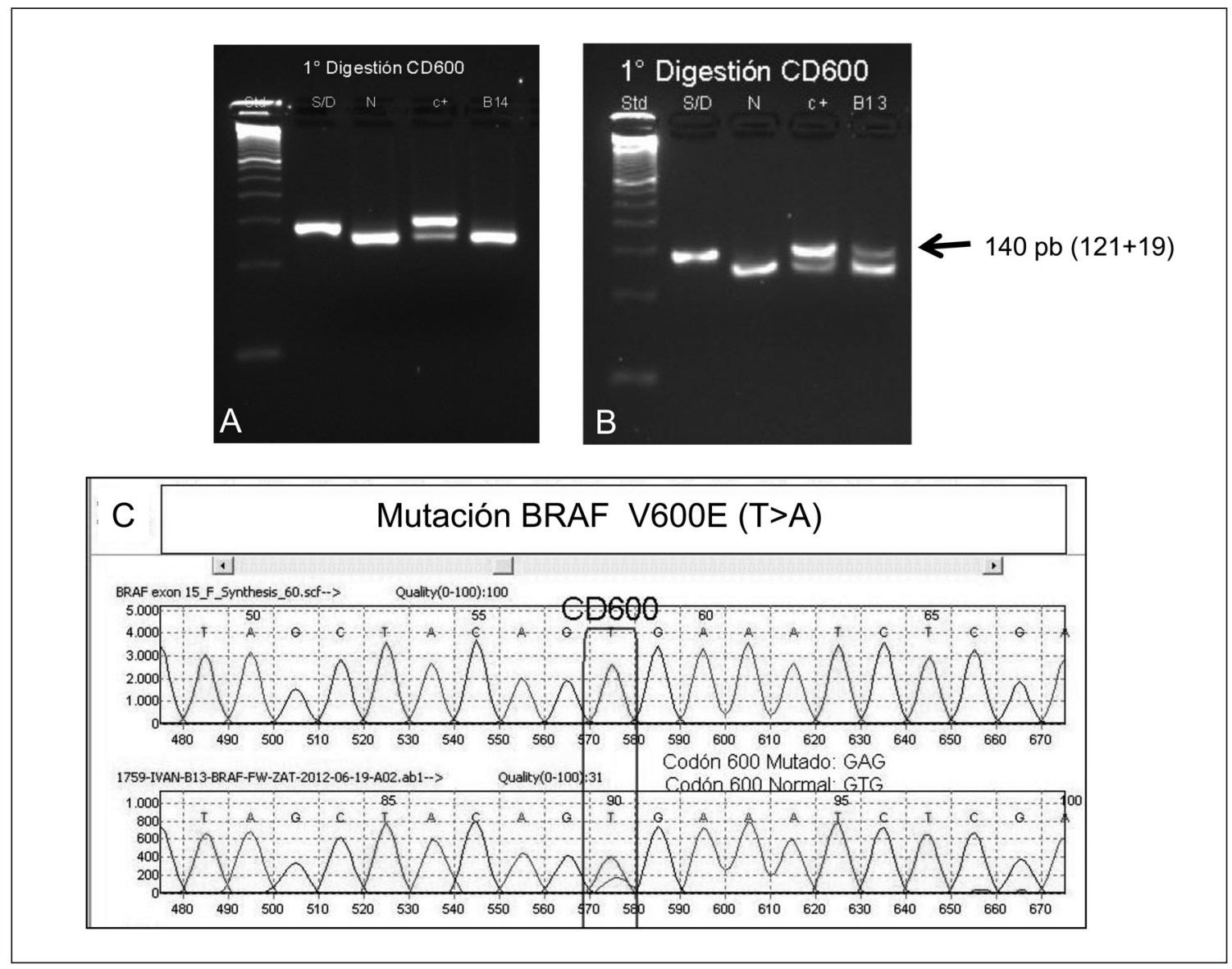

Figura 1. 


\section{Secuenciación}

Los productos amplificados fueron purificados en columnas Qiagen, (Quiaquick. PCR purification kit) y junto con el partidor reverso fueron secuenciados en equipo ABI PRISM 3100 Applied Biosystems.

\section{Controles}

Como control positivo, se empleó $\mathrm{ADN}$ de carcinomas papilares del tiroides y melanomas en los que previamente se había demostrado la mutación del BRAF en el codón 15. Como control negativo se usó ADN genómico comercial (Promega) y como control blanco se sustituyó el ADN por agua deionizada.

\section{Análisis estadístico}

Se realizó mediante test de $\chi^{2}$ y exacto de Fisher para las tablas de contingencia y t-test para los promedios de muestras independientes.

\section{Resultados}

El grupo total estaba constituido por 100 casos, 56 casos correspondieron a mujeres con un promedio de edad fue de 61,1 años (DS $\pm 13,5$ años) y los restantes 44 casos a hombres con un promedio de edad de 61,8 años (DS $\pm 10,5$ años). La totalidad de los casos correspondieron a adenocarcinomas. Las características generales del grupo estudiado se muestran en la Tabla 1. Los tumores primarios se

Tabla 1. Características generales

\begin{tabular}{|lrcc|}
\hline Género & n & $\begin{array}{c}\text { Mujeres } \\
\text { Edad (años) }\end{array}$ & $\begin{array}{c}\text { Hombres } \\
\text { DS }\end{array}$ \\
Mujeres & & & \\
Hombres & 56 & 61,1 & 13,5 \\
Localización & 44 & 61,8 & 10,5 \\
Colon & 88 & 49 & \\
Recto & 6 & 3 & 39 \\
Metástasis & 5 & 3 & 3 \\
No precisado & 1 & 1 & 0 \\
Mutación & & & \\
KRAS mutado & 42 & 22 & 20 \\
BRAF mutado & 12 & 7 & 5 \\
No mutado & 46 & 27 & 19 \\
Total & 100 & 56 & 44 \\
\hline
\end{tabular}

localizaron en colon en 88 casos en colon, 6 casos en recto y 5 casos correspondieron a metástasis hepáticas. No se logró precisar la localización del tumor en sólo 1 caso. En 12 de los 58 casos (20,1\%) que fueron estudiados, se demostró mutación del gen BRAF, los 46 casos restantes fueron de tipo silvestre o no mutado. Por lo tanto, la frecuencia de mutaciones para BRAF en este grupo de pacientes sería de $12 \%$. Todas las mutaciones comprometieron al codón 15 en la posición nucleotídica 1796 V600E (Figura 1c). No se observaron diferencias entre la frecuencia de mutaciones de BRAF y el género de los pacientes ( 7 mujeres y 5 hombres). En 11 de 94 casos (12\%) de los tumores primarios del colon y recto presentaron mutación de Braf y sólo 1 de 5 metástasis (20\%). Sin embargo, esta diferencia y el escaso número de observaciones no permitió establecer diferencias $(\mathrm{p}=\mathrm{ns})$. De esta manera, la frecuencia de mutaciones para la vía RAS-RAF fue en conjunto de $54 \%$ en el cáncer de colon y recto en esta serie.

\section{Discusión}

Nuestros hallazgos muestran la presencia de mutación del gen BRAF estaría presente en $12 \%$ de los cánceres de colon y recto. Debemos considerar que a este grupo de pacientes inicialmente se les realizó estudio de la mutación del gen KRAS, el cual resultó ser positivo en $42 \%$. Estas cifras son similares a las reportadas en la literatura respecto de ambos genes ${ }^{24,31}$, por lo cual, la frecuencia acumulativa de mutación de KAS y BRAF es de 54\% en nuestro medio, cifra que podría aumentar levemente si agrega el estudio de mutaciones de otros codones de menor frecuencia del gen KRAS como 61 y 146, entre otros. Estos resultados sugieren que la terapia con anticuerpos monoclonales contra EGFR en el cáncer de colon y recto avanzado no tendría indicación en este grupo de pacientes, ya que ambos eventos serían un predictor de resistencia en más de la mitad de los pacientes.

En la actualidad, algunas recomendaciones para el cáncer de colon y recto avanzado sugieren establecer el status de KRAS en forma inicial y continuar con la determinación de BRAF en el caso que el primero fuese negativo ${ }^{32-34}$. Otros genes que pudiesen participar en predecir resistencia a la terapia anti EGFR como los de la vía PIP3KAKT-mTOR, están en estudio ${ }^{35}$. También se han 
demostrado diferencias en la frecuencia de mutaciones de BRAF en distintas etnias, por lo que sería importante determinar su status en nuestra población originaria $^{36,37}$.

Respecto de las técnicas empleadas en este estudio como el RFLP y secuenciación directa, obtuvimos la misma conclusión que el trabajo realizado con el gen KRAS. La técnica de RFLP mostró una concordancia en $100 \%$ de los casos con la secuenciación directa, lo cual dada su sencillez y facilidad de interpretación, abre las posibilidades de contar con estas determinaciones en los Servicios de Anatomía Patológica de centros asistenciales públicos ${ }^{38}$.

Es altamente probable que la próxima clasificación del cáncer de colon y recto incluya la determinación de una serie de marcadores moleculares, tanto como factores pronósticos y predictivos de respuesta a terapia dirigida. Entre los genes candidatos a esta clasificación se encuentran además de los estudiados en este trabajo, el estudio de la inestabilidad microsatelital, p53 y PIP3k entre los más frecuentemente señalados por los estudios publicados ${ }^{39}$. De esta manera, la implementación de estas determinaciones son una necesidad actual y un elemento fundamental en la elección de la terapia dirigida a los portadores de esta frecuente enfermedad tumoral.

\section{Referencias}

1. The International Agency for Research on Cancer. Globocan. http://www.iarc.fr/en/media-centre/iarcnews/2011/globocan2008-prev.php 2008.

2. http://deis.minsal.cl/index.asp. 2012.

3. Zárate AJ, Alonso FT, Garmendia ML, López-Kostner F. Increasing crude and adjusted mortality rates for colorectal cancer in a developing South American country. Colorectal Dis 2013; 15 (1): 47-51.

4. (http://deis.minsal.cl/vitales/vitales2010/Serie_Mortalidad_causa_Chile_2000_2010/Chile.htm).2010.

5. Bacolod MD, Barany F. Molecular profiling of colon tumors: the search for clinically relevant biomarkers of progression, prognosis, therapeutics, and predisposition. Ann Surg Oncol 2011; 18 (13): 3694-700.

6. Fearon ER. Molecular genetics of colorectal cancer. Annu Rev Pathol 2011; 6: 479-507.

7. Wee S, Jagani Z, Xiang KX, Loo A, Dorsch M, Yao YM, et al. PI3K pathway activation mediates resistance to MEK inhibitors in KRAS mutant cancers. Cancer Res 2009; 69

\section{(10): 4286-93.}

8. Beerenwinkel N, Antal T, Dingli D, Traulsen A, Kinzler KW, Velculescu VE, et al. Genetic progression and the waiting time to cancer. PLoS Comput Biol 2007; 3 (11): e225.

9. Frattini M, Balestra D, Suardi S, Oggionni M, Alberici P, Radice P, et al. Different genetic features associated with colon and rectal carcinogenesis. Clin Cancer Res 2004; 10 (12 Pt 1): 4015-21.

10. Jones S, Chen WD, Parmigiani G, Diehl F, Beerenwinkel $\mathrm{N}$, Antal T, et al. Comparative lesion sequencing provides insights into tumor evolution. Proc Natl Acad Sci USA 2008; 105 (11): 4283-8.

11. Lan YT, Yang SH, Chang SC, Liang WY, Li AF, Wang HS, et al. Analysis of the seventh edition of American Joint Committee on colon cancer staging. Int J Colorectal Dis 2012; 27 (5): 657-63.

12. Di Nicolantonio F, Martini M, Molinari F, SartoreBianchi A, Arena S, Saletti P, et al. Wild-type BRAF is required for response to panitumumab or cetuximab in metastatic colorectal cancer. J Clin Oncol 2008; 26 (35): 5705-12.

13. Allegra CJ, Jessup JM, Somerfield MR, Hamilton SR, Hammond EH, Hayes DF, et al. American Society of Clinical Oncology provisional clinical opinion: testing for KRAS gene mutations in patients with metastatic colorectal carcinoma to predict response to anti-epidermal growth factor receptor monoclonal antibody therapy. J Clin Oncol 2009; 27 (12): 2091-6.

14. Petrelli F, Borgonovo K, Cabiddu M, Ghilardi M, Barni S. Cetuximab and panitumumab in KRAS wild-type colorectal cancer: a meta-analysis. Int J Colorectal Dis 2011; 26 (7): 823-33.

15. Valtorta E, Misale S, Sartore-Bianchi A, Nagtegaal ID, Paraf F, Lauricella C, et al. KRAS gene amplification in colorectal cancer and impact on response to EGFRtargeted therapy 10.1002/ijc.28106. Int J Cancer 2013.

16. Yokota T. Are KRAS/BRAF mutations potent prognostic and/or predictive biomarkers in colorectal cancers? Anticancer Agents Med Chem 2012; 12 (2): 163-71.

17. Dahabreh IJ, Terasawa T, Castaldi PJ, Trikalinos TA. Systematic review: Anti-epidermal growth factor receptor treatment effect modification by KRAS mutations in advanced colorectal cancer. Ann Intern Med 2011; 154 (1): 37-49.

18. Sartore-Bianchi A, Di Nicolantonio F, Nichelatti M, Molinari F, De Dosso S, Saletti P, et al. Multi-determinants analysis of molecular alterations for predicting clinical benefit to EGFR-targeted monoclonal antibodies in colorectal cancer. PLoS One 2009; 4 (10): e7287.

19. Yuen ST, Davies H, Chan TL, Ho JW, Bignell GR, Cox 
C, et al. Similarity of the phenotypic patterns associated with BRAF and KRAS mutations in colorectal neoplasia. Cancer Res 2002; 62 (22): 6451-5.

20. Wang L, Cunningham JM, Winters JL, Guenther JC, French AJ, Boardman LA, et al. BRAF mutations in colon cancer are not likely attributable to defective DNA mismatch repair. Cancer Res 2003; 63 (17): 5209-12.

21. Deng G, Bell I, Crawley S, Gum J, Terdiman JP, Allen BA, et al. BRAF mutation is frequently present in sporadic colorectal cancer with methylated hMLH1, but not in hereditary nonpolyposis colorectal cancer. Clin Cancer Res 2004; 10 (1 Pt 1): 191-5.

22. Li X, Yao X, Wang Y, Hu F, Wang F, Jiang L, et al. MLH1 Promoter Methylation Frequency in Colorectal Cancer Patients and Related Clinicopathological and Molecular Features. PLoS One 2013; 8 (3): e59064.

23. Sahin IH, Kazmi SM, Yorio JT, Bhadkamkar NA, Kee BK, Garrett CR. Rare Though Not Mutually Exclusive: A Report of Three Cases of Concomitant KRAS and BRAF Mutation and a Review of the Literature. J Cancer 2013; 4 (4): 320-2.

24. Pakneshan S, Salajegheh A, Smith RA, Lam AK. Clinicopathological relevance of BRAF mutations in human cancer. 10.1097/PAT.0b013e328360b61d. Pathology 2013.

25. Rajagopalan H, Bardelli A, Lengauer C, Kinzler KW, Vogelstein B, Velculescu VE. Tumorigenesis: RAF/RAS oncogenes and mismatch-repair status. Nature 2002; 418 (6901): 934.

26. Govender D, Chetty R. Gene of the month: BRAF. J Clin Pathol 2012; 65 (11): 986-8.

27. Cantwell-Dorris ER, O'Leary JJ, Sheils OM. BRAFV600E: implications for carcinogenesis and molecular therapy. Mol Cancer Ther 2011; 10 (3): 385-94.

28. Dienstmann R, Tabernero J. BRAF as a target for cancer therapy. Anticancer Agents Med Chem 2011; 11 (3): 28595.

29. Loupakis F, Ruzzo A, Cremolini C, Vincenzi B, Salvatore L, Santini D, et al. KRAS codon 61, 146 and BRAF mutations predict resistance to cetuximab plus irinotecan in KRAS codon 12 and 13 wild-type metastatic colorectal cancer. Br J Cancer 2009; 101 (4): 715-21.
30. Laurent-Puig P, Cayre A, Manceau G, Buc E, Bachet JB, Lecomte T, et al. Analysis of PTEN, BRAF, and EGFR status in determining benefit from cetuximab therapy in wild-type KRAS metastatic colon cancer. J Clin Oncol 2009; 27 (35): 5924-30.

31. Phipps AI, Buchanan DD, Makar KW, Win AK, Baron JA, Lindor NM, et al. KRAS-mutation status in relation to colorectal cancer survival: the joint impact of correlated tumour markers. 10.1038/bjc.2013.118. Br J Cancer 2013.

32. Akiyoshi T, Kobunai T, Watanabe T. Recent approaches to identifying biomarkers for high-risk stage II colon cancer. Surg Today 2012; 42 (11): 1037-45.

33. Muhammad S, Jiang Z, Liu Z, Kaur K, Wang X. The role of EGFR monoclonal antibodies (MoABs) cetuximab/ panitumab, and BRAF inhibitors in BRAF mutated colorectal cancer. J Gastrointest Oncol 2013; 4 (1): 72-81.

34. Fleming M, Ravula S, Tatishchev SF, Wang HL. Colorectal carcinoma: Pathologic aspects. J Gastrointest Oncol 2012; 3 (3): 153-73.

35. Bardelli A, Siena S. Molecular mechanisms of resistance to cetuximab and panitumumab in colorectal cancer. 2009.24.6116. J Clin Oncol 2010; 28 (7): 1254-61.

36. English DR, Young JP, Simpson JA, Jenkins MA, Southey MC, Walsh MD, et al. Ethnicity and risk for colorectal cancers showing somatic BRAF V600E mutation or CpG island methylator phenotype. Cancer Epidemiol Biomarkers Prev 2008; 17 (7): 1774-80.

37. Chaiyapan W, Duangpakdee P, Boonpipattanapong T, Kanngern S, Sangkhathat S. Somatic Mutations of K-Ras and BRAF in Thai Colorectal Cancer and their Prognostic Value. Asian Pac J Cancer Prev 2013; 14 (1): 329-32.

38. Anderson SM. Laboratory methods for KRAS mutation analysis. Expert Rev Mol Diagn 2011; 11 (6): 635-42.

39. Calonge N, Fisher NL, Berg AO, Campos-Outcalt D, Djulbegovic B, Ganiats TG, et al. Recommendations from the EGAPP Working Group: can testing of tumor tissue for mutations in EGFR pathway downstream effector genes in patients with metastatic colorectal cancer improve health outcomes by guiding decisions regarding anti-EGFR therapy? 10.1038/gim.2012.184. Genet Med 2013. 